\title{
La política social como instrumento para la producción de información
}

\author{
Mora Straschnoy \\ Universidad de Buenos Aires (UBA)
}

\author{
Mora Kantor \\ Universidad de Buenos Aires (UBA)
}

\section{La política social como instrumento para la producción de información}

Resumen: El artículo tiene dos objetivos. El primero es analizar los déficits del Estado argentino en materia de producción de información técnica ambiental. El segundo, es estudiar la utilización del andamiaje de una política de transferencia de ingresos como la Asignación Universal por Hijo, para producir información e implementar controles sanitarios en una población determinada. Para estos fines se analiza un convenio de colaboración entre la Administración Nacional de la Seguridad Social (Anses) y la Autoridad de Cuenca Matanza Riachuelo (Acumar) realizado en el marco de lo ordenado por la Corte Suprema de Justicia de la Nación en la Causa Mendoza. Palabras clave: Producción de información pública. Condicionalidades. Cuenca Matanza Riachuelo. Activismo judicial Asignación Universal por Hijo.

\section{A política social como instrumento para a produção da informação}

Resumo: O presente artigo tem dois objetivos. O primeiro é analisar os déficits do Estado Argentino em matéria de produção da informação técnico-ambiental. O segundo seria estudar a utilização da estrutura de uma política de transferência de ingressos como a Atribuição Universal por Filho, para produzir informação e programar controles sanitários em uma determinada população. Portanto, se analisa um convênio de colaboração entre a Administração Nacional da Segurança Social (Anses) e a Autoridade da Cuenca Matanza Riachuelo (Acumar) que foi realizado no marco do ordenado pela Corte Suprema de Justiça da Nação na Causa Mendoza.

Palavras-chave: Produção da informação pública. Condicionalidades. Cuenca Matanza Riachuelo. Ativismo judicial. Atribuição Universal por Filho.

\section{Social Policy as an Instrument for the Production of Information}

Abstract: This article has two objectives. The first is to analyze diificulties the Argentine state has in relation to the production of technical information about the environment. The second is to study the use of the framework of an income transfer policy such as the Universal Allowance per Child, for producing information and implementing sanitary control for a certain population. For these purposes it analyzes a collaboration contract between the National Social Security Administration (Anses) and the Authority of Cuenca Matanza Riachuelo (Acumar) conducted as the result of a decision by the national Supreme Court referring to Mendoza. Keywords: Production of public information. Conditionalities. Cuenca Matanza Riachuelo. Judicial activism. Universal. 


\section{Introducción}

Este artículo analiza un convenio de colaboración entre dos organismos pertenecientes a la rama ejecutiva del Estado argentino - la Administración Nacional de la Seguridad Social (Anses) y la Autoridad de Cuenca Matanza Riachuelo (Acumar) ${ }^{1}$. El convenio firmado en el año 2012 formaliza la implementación de una encuesta socioambiental a los receptores de la Asignación Universal por Hijo para Protección Social - de aquí en más AUH o Asignación. LaAsignación creada en 2009 constituye una de las políticas sociales no contributivas más importantes por su alcance (más de 3,3 millones de niños, niñas y adolescentes), el valor de la transferencia mensual ( $\$ 460$ U\$S83) y su inscripción institucional dentro del Sistema de Asignaciones Familiares, propio de la Seguridad Social ${ }^{2}$. La AUH contempla condicionalidades en materia de salud y educación, en función de las cuales se retiene el $20 \%$ de la prestación hasta que se acredite anualmente su cumplimiento ante las oficinas de la Anses.

El objetivo de la encuesta es producir información sobre la exposición de la población, menor a 6 años que habita en la zona de influencia de la Cuenca Matanza Riachuelo (CMR), a factores de riesgo, e identificar patologías como consecuencia de la situación ambiental. La cuenca constituye una de las más contaminadas del mundo, y ahí radica la importancia de conocer el estado de la salud de la población que allí habita.

Las características, objetivos y el contexto de surgimiento de este convenio inter-organismos que se da hacia el interior del entramado estatal, da el puntapié para abordar dos cuestiones de gran relevancia en el universo de las políticas públicas: la dimensión informacional del proceso de formulación de políticas y las condicionalidades en las políticas sociales.

Con relación al primero de los puntos marcados, el caso bajo la lupa ilustra los déficits del Estado argentino en materia de producción de información. Las carencias no solo se observan al momento de poner en marcha un programa de política pública - en este caso ambiental -, cuando se torna evidente que los organismos a cargo de la formulación de la política no cuentan con los datos y la información necesaria para abordar la problemática objetivo, sino que también en el proceso de confección e implementación del programa que se quiere aplicar, al no preverse en la arquitectura del mismo, instancias de relevamiento de datos, sistematización y análisis de información como parte constitutiva de la política. En otras palabras, no solo no hay información disponible a priori sino que el componente informacional parece no tener un lugar relevante en la formulación de la política ${ }^{3}$.

Por ello, las iniciativas para producir información son decididas y diseñadas “sobre la marcha”. Lejos de constituir parte de una estrategia integral, son intervenciones ad-hoc, cuyo devenir errático atenta contra la calidad de los datos que se relevan y la información que se produce con esos datos. La encuesta socioambiental que aquí se estudia constituye un ejemplo de lo antedicho. El contexto de su surgimiento, las decisiones en torno a la población a encuestar y los dispositivos elegidos para su implementación así lo demuestran.

El análisis del convenio a su vez interpela y motoriza preguntas y reflexiones sobre la AUH, tanto sobre su diseño, como en referencia a los supuestos sobre la población receptora. La encuesta se convierte en una nueva exigencia para quienes perciben esta política, que no pueden elegir si participar o no de la estrategia de recolección de información - a diferencia de lo que ocurre con otras iniciativas que implementa la Acumar. La obligatoriedad de participar de la encuesta no es explicita, pero en la práctica resulta evidente al montarse sobre procedimientos establecidos para que los receptores de la AUH acrediten el cumplimento de las condicionalidades. ¿En qué se funda este trato desigual por parte del Estado?

Luego de esta introducción, el artículo se organiza de la siguiente manera: en la segunda sección, se enmarca el surgimiento del convenio en la causa "Mendoza" a través de un recorrido por los fallos de la Corte Suprema Argentina en este caso de litigio estructural, y se analiza el desempeño del Estado en materia de relevamiento y producción de información pública. En la tercera sección, se describe brevemente las características del Convenio. En la cuarta, el artículo intenta posicionar a la encuesta socio-ambiental y los controles de salud que de ella derivan en el mundo de las condicionalidades; al tiempo que se pone en relieve una serie de tensiones y nuevos universos de preguntas que se abren en el marco de las vinculaciones que produce el convenio. Finalmente, en el último apartado, se esbozan las principales conclusiones.

La metodología utilizada es cualitativa. La elaboración del artículo se baso en un análisis normativo, en respuestas estatales a pedidos de información pública ${ }^{4}$, y en una entrevista a un funcionario de la Acumar y otra al director de una organización civil especializada en temas ambientales y parte litigante del Caso "Mendoza".

\section{El contexto de surgimiento de la Encuesta socio-ambiental y los déficits del Estado argentino en materia de producción de información}

El Convenio bajo análisis se enmarca en la causa judicial más importante en temas ambientales en la Argentina, la causa "Mendoza". Ésta se inicia en el año 2006 cuando un grupo de vecinos y vecinas afectados 
por la situación ambiental en la Cuenca Matanza-Riachuelo presentan una demanda contra un número de empresas radicadas en la zona y contra los poderes ejecutivos del gobierno Nacional, el de la Provincia de Buenos Aires, y el de la Ciudad de Buenos Aires.

La encuesta constituye una iniciativa de relevamiento de datos que los organismos convenientes ponen en práctica para cumplir con una de las tantas exigencias que, en materia de producción y acceso a la información pública, la Corte Suprema Argentina ordena a través de los distintos fallos que componen esa causa ${ }^{5}$. Las políticas que la Corte ordena debían cumplir con tres objetivos claves: mejorar la calidad de vida de los habitantes de la cuenca; recomponer el ambiente de la cuenca en todos sus componentes (agua, aire y suelo); y prevenir daños con suficiente y razonable grado de predicción.

Además de impulsar la creación de un organismo específico a cargo de la formulación de un plan integral (la Acumar), las sentencias del máximo Tribunal pusieron en funcionamiento la maquinaria estatal de producción de políticas públicas, poniendo fin al estancamiento que impedía solucionar el problema de contaminación en la CMR, el que databa de hace más de 200 años.

Esta es una de las razones por las que este fallo constituye un caso paradigmático. Se trata de una de las primeras veces que la Corte, en pos de remediar los daños causados por la vulneración del derecho colectivo a un ambiente sano, se involucra en el proceso de formulación de políticas al ordenar a los poderes ejecutivos el diseño y la implementación de un plan integral de saneamiento. En este marco de activismo judicial temática que reviste gran actualidad en la Argentina y en términos generales en Latinoamérica -, uno de los puntos especialmente relevantes a los fines de este artículo es el lugar central que la Corte asigna a uno de los componentes fundamentales de todo proceso de hechura de políticas: el componente de relevamiento, sistematización, utilización de y acceso a información de interés para el Estado y para los ciudadanos.

La Corte Suprema de Justicia de la Nación Argentina emite fallos en los años 2006, 2007, 2008 y 2010. El análisis cronológico de estas sentencias deja entrever una preocupación incremental con relación a la necesidad de contar con información adecuada sobre la situación ambiental de la cuenca, sobre el estado de salud de los vecinos que habitan sus inmediaciones, así como sobre las características de las empresas radicadas en las zonas aledañas a la misma.

En este sentido, la Corte trasciende el "tratamiento jurídico" del componente informacional al no abordar solamente la problemática desde la óptica de la información en tanto derecho - es decir el derecho fundamental de toda persona a acceder a información en manos del Estado ${ }^{6}$ - sino al enfatizar la importancia de contar con información adecuada para poder diseñar programas de política pública racionales y eficaces.

Ya en el primero de sus fallos, en el año 2006, la Corte hace hincapié en la falta de información en materia ambiental. Así, en este fallo, la Corte, señalaba que "no existe la información adecuada, ya que la demanda no ilustra al tribunal aspectos esenciales sobre la cuestión litigiosa" (FALLO MENDOZA, 2006, consid. 19). Además, ya señalando el carácter vetusto de la información que hasta ese momento existía sobre el estado de la situación ambiental en la CMR, afirmaba Fallo Mendoza (2006, consid. 11) que "el escrito introductorio tampoco se basa en estudios actualizados, ya que se remite a publicaciones periodísticas o a informes presentados por diversos organismos hace varios años".

La Corte responsabiliza, desde un principio, al Estado por la falta de información adecuada y actualizada, tanto en los procesos de producción de información como en los mecanismos de acceso a esa información. Por ello desde el inicio le ordena a los poderes ejecutivos producir información y hacerla pública. Las partes demandadas deben presentar un plan integrado, el cual debe contemplar no solo la elaboración de un estudio de impacto ambiental de las empresas involucradas en la demanda, sino también la implementación de un "programa de información ambiental pública a todo el que la requiera, especialmente los ciudadanos del área territorial involucrada" (FALLO MENDOZA, 2006, consid. 5).

Es decir que, ya desde el primero de sus fallos, la Corte se encarga de abordar las dos partes de la compleja e inevitable díada: producción de y acceso a información pública. Producción y acceso se entrelazan y confunden, pero son cuestiones diferentes. Un tema es que el Estado produzca información, qué información produce y de qué calidad es, y otro muy distinto es que la información que el Estado produce sea de acceso público a través de procedimientos especialmente establecidos para ello.

En la medida en que la Corte va advirtiendo, fallo tras fallo, las carencias de los poderes ejecutivos en relación con la información con la que cuentan va tornando más especificas las órdenes sobre qué información es la que se debe producir.Es decir, el Tribunal no sólo obliga a los Estados a elaborar estudios e informes sino que toma decisiones sobre sus contenidos, estableciendo qué datos se deben relevar y qué información se debe producir.

Estos lineamientos le otorgan más peso a lo que puede leerse como el involucramiento del máximo Tribunal en el proceso de formulación de políticas; toda vez que se entiende que las decisiones acerca de qué información debe producirse no son puramente técnicas, sino que refieren a la forma de entender el problema 
público que se quiere abordar y establecen cuáles serán las pautas para su solución, y hasta qué valores o principios éticos subyacen a las decisiones de política pública.

Por su parte, para entender el por qué de los avances de la Corte en materia informacional, es imprescindible hacer referencia a dos hitos que tuvieron lugar en el transcurso de la causa. Uno es la presentación por parte de la Secretaría de Ambiente de la Nación de un "Plan Integral de Saneamiento de la cuenca MatanzaRiachuelo" en el marco de lo requerido por el alto Tribunal en el primer fallo del año 2006. El segundo evento importante es la elaboración de un informe de peritaje realizado por expertos de la Universidad de Buenos Aires (UBA), en el año 2007, sobre dicho Plan Integral; también por encargo de la Corte Suprema. Los profesionales a cargo de la redacción del informe detectaron deficiencias tanto en la información y los indicadores sobre los que se basa el proyecto, como en las acciones propuestas.

Las debilidades halladas explican gran parte del contenido de los fallos posteriores al año 2007. Los resultados del informe constituyen una de las razones por las cuales la Corte va ordenando requerimientos cada vez más específicos en materia de producción de información como, por ejemplo, la adopción de un sistema internacional de medición del nivel de cumplimiento de los objetivos establecidos que ordena en el fallo del año 2008 (FALLO MENDOZA, 2006, consid. 17).

El trabajo de la UBA no solo marca la falta concreta de datos vinculados con cuestiones sanitarias en la zona de influencia de la cuenca (advierte "que no hay datos sobre enfermedades prevalentes, cantidad de población afectada, atención de casos e indicadores de riesgo de exposición" (UBA, 2007), sino que pone luz sobre un tipo de déficit informacional más estructural, intrínseco a la forma del Estado argentino de elaborar programas y políticas públicas. Las críticas del informe ponen de relieve un problema que estudios posteriores y más profundos podrán reafirmar. Al momento de formular la política, los tomadores de decisiones no prevén ni incluyen instancias o herramientas de producción, sistematización y análisis de información pertinente para el diseño, la implementación y la evaluación de la propia política en ejecución. Ello no solo repercute en el desempeño de ese programa o política en particular, sino que impacta de forma negativa en el sistema de elaboración de políticas en su conjunto ya que no se acumula información que luego pueda ser utilizada en otras políticas, incluso pertenecientes a otros campos.

El informe vaticina el fracaso del plan de saneamiento presentado por la Acumar, toda vez que - al no incluir las instancias previas de producción de información - no cuenta con los elementos básicos y necesarios para analizar la situación de la salud y sus condicionantes, identificar los problemas sanitarios prioritarios, definir los objetivos de las intervenciones y evaluar el logro de los objetivos. Ningún especialista en administración y políticas públicas desconoce que los estudios de línea de base, la identificación de los principales problemas a abordar, el establecimiento de objetivos y su monitoreo y evaluación, son momentos básicos y constitutivos de cualquier proceso de formulación de políticas públicas. El caso "Mendoza", observado tras la lente de los estudios sobre la confección de políticas públicas, constituye un ejemplo de cómo los sistemas de información

El debate sobre las condicionalidades gira entorno a la legitimidad, la razonabilidad y la eficiencia de tal herramienta para garantizar la satisfacción del derecho a la educación y salud. y los procesos de su producción no suelen constituir parte de la hechura de la política, sino que se piensan y diseñan "por fuera" de la política y a través de mecanismos ad-hoc cuando quienes ejecutan la políticas se topan con lagunas informacionales. Ello, muchas veces redunda en falta de oportunidad de la información que se produce (el Estado carece de la información adecuada en cada uno de los momentos de intervención sobre el territorio) y falta de pertinencia (los mecanismos de producción de información que se montan no están diseñados desde el corazón de la política ni están liderados por especialistas, por lo que la información que se releva termina no siendo la adecuada para resolver los problemas que originaron la intervención del Estado desde un comienzo).

La encuesta que surge del convenio Anses-Acumar es un ejemplo más de la lógica ad-hoc de producción de información pública arriba descrita, pero a su vez, presenta una particularidad que la vuelve muy rica para el análisis: se monta sobre una política social, la AUH. Esta interrelación entre la necesidad de la Acumar de producir información pública y los mecanismos de funcionamiento de la $\mathrm{AUH}$, motoriza indagaciones sobre el diseño de la política social, y sobre los supuestos y concepciones que recaen sobre sus receptores, que serán de interés abordar a continuación. 


\section{Características y alcances del Convenio}

Tal como se dijo anteriormente, el convenio bajo análisis establece la encuesta como un paso necesario para finalizar el trámite anual que realizan los receptores de la AUH con objeto de certificar las condicionalidades. Aunque no figura en el documento de creación de la encuesta, a través de una entrevista con un funcionario de alto-rango de la Acumar se supo que la idea del organismo es, en una segunda instancia, exigir la realización de una serie de chequeos médicos a los receptores de la AUH que en sus respuestas arrojen datos que demuestren que están expuestos a factores de riesgo. El cumplimiento de esos chequeos será condicionante para continuar recibiendo la AUH.

Es decir que el convenio produce dos instancias que son importes de diferenciar, y que corresponde analizar separadamente. La primera, refiere a la contestación de la encuesta al momento de acreditar ante la Anses el cumplimiento de las condicionalidades. Esta encuesta tiene como objetivo "contribuir a la identificación de patologías vinculadas a factores de carácter ambiental y posibilitar su prevención y tratamiento" (ARGENTINA, 2012, p. 3). La segunda instancia tiene como objetivo que los receptores de la AUH que en la encuesta revelen que se encuentran bajo un riesgo potencial, se sometan a exámenes médicos.

En primer término en lo que refiere a las indagaciones y debates que el convenio motoriza, interesará analizar si la forma en la que se operacionaliza el convenio redunda en una nueva condicionalidad o en una exigencia de otro tipo, y si la definición es unánime para ambas instancias descritas o se pueden establecer diferencias.

El análisis del carácter condicionante del convenio no se explica por una curiosidad ontológica, sino que resulta de interés en la medida en que se asocia con nuevas demandas sobre una política, la AUH, ya en práctica. En este sentido aparece la pregunta por la legitimidad de esta nueva exigencia, y la necesidad de estudiar cuáles son sus objetivos y mecanismos puestos a disposición para lograrlos.

En relación con la definición de las condicionalidades, su alcance y límites, en la bibliografía latinoamericana especializada no existe consenso sobre el significado del término. Esto no ocurre porque haya definiciones en disputa sino porque no se encuentra de forma generalizada un esfuerzo por definir el concepto en uso ${ }^{7}$. La definición de las condicionalidades parece surgir más de su uso práctico que de una convención conceptual. Por este motivo, el objetivo del próximo apartado es rastrear estos usos a fin de poder producir una definición que permita utilizar con sustento el concepto, y dar respuesta a interrogantes del tipo: ¿Cuál es el límite entre una condicionalidad y una exigencia de otro tipo en las políticas de transferencia? ¿Cuál es la particularidad del concepto "condicionalidades”? ¿Es éste propio de los PTC y las políticas similares que los trascendieron, o puede encontrarse en otros ámbitos de aplicación? El dilucidar estos interrogantes permitirá caracterizar a la encuesta socio-ambiental y explicar su lugar dentro del universo de la condicionalidades.

\section{Origen y definición de las Condicionalidades}

En los primeros años del presente siglo se comienzan a implementar en América Latina políticas destinadas a familias calificadas como pobres y/o vulnerables cuyo objetivo es transferir ingresos con la condicionalidad de que las mismas cumplan con ciertas exigencias, generalmente relacionadas con prácticas educativas y/o sanitarias.

Aquí surge la pregunta sobre si la definición de las condicionalidades se establece por la exigencia en sí, más allá de cual sea su objetivo o si el desarrollo del capital humano (a través de mejorar la salud y la educación) es el elemento determinante. Si la respuesta fuera la primera opción, entonces la encuesta resultante del convenio Acumar-Anses podría ser entendida como una condicionalidad, aún cuando ésta tuviera como único objetivo brindar al Estado datos primarios para la producción de información. La situación sería la inversa si al finalizar el presente desarrollo analítico se opta por una definición sustantiva de las condicionalidades. En tal caso toda exigencia que no tenga como propósito directo promover el desarrollo del capital humano de los receptores de las políticas, debería ser considerada como una exigencia, parte del diseño de la política, pero no como una condicionalidad.

Cuando se analizan los casos empíricos de políticas condicionadas más importantes de Latinoamérica, éstas suelen estar relacionadas con el incremento del capital humano y el cumplimiento de derechos. Principalmente, las condicionalidades están relacionadas con el desarrollo del capital humano vía el aumento de los controles de salud y la asistencia a establecimientos educativos.

Estas observaciones permiten arribar a una definición común: se hará uso del concepto de condicionalidades cuando las exigencias en función de las que se condicionen las transferencias tengan como objetivo explícito e inmediato el desarrollo del capital humano de quienes se encuentran implicados en la política. Es decir, se opta por el uso sustantivo del concepto. Como resultado de ésta conclusión, se 
deduce que la exigencia de la encuesta que aquí se estudia no constituye en sí misma una condicionalidad, sino que una exigencia de otro tipo, mientras que la obligación para quienes tuvieran resultados negativos en la encuesta de realizarse los chequeos médicos sí lo es; en tanto los mismos tienen el objetivo del bienestar físico de los directamente implicados en la política.

Definido el concepto de condicionalidades en términos generales y, en relación con el convenio en particular, interesa analizara las particularidades de éste debate en relación a las implicancias del convenio.

\section{La inscripción del Convenio en el debate sobre las condicionalidades}

El debate sobre las condicionalidades gira entorno a la legitimidad, la razonabilidad y la eficiencia de tal herramienta para garantizar la satisfacción del derecho a la educación y salud. Se trata de un debate no resuelto, que en el caso concreto bajo análisis, presenta una particularidad de magnitud: las condicionalidades surgidas del convenio no han sido puestas en uso para el cumplimiento de derechos/obligaciones exigidos por otras políticas universales, sino para la realización de exámenes de salud específicos por la cuestión de la CMR.

El análisis se complejiza aún más al observar que la propia Acumar realiza en algunos barrios y villas otros operativos territoriales para relevar información sanitaria en el marco de la Evaluación Integral de Salud en Áreas de Riesgo (Eisar). Pero en ese caso específico, a diferencia de la realización de la encuesta que aquí se estudia, las familias deben firmar un consentimiento para que la salud de los niños pueda ser evaluada. Poniendo en suspenso por cuestiones de espacio el extenso debate teórico-filosófico sobre la potestad del Estado para imponer prácticas sanitarias a sus ciudadanos, interesa evidenciar que se le exige a los receptores de la AUH lo que al resto de la población se le ofrece como opción. Sin poner en cuestión las "buenas intenciones" estatales detrás de la exigencia de chequeos médicos, no se puede dejar de señalar el trato desigual.

Encender esta luz roja resulta de interés en la medida de que cristaliza el hecho de que cuando el Estado actúa en pos del "bienestar de la población", generalmente calificada como vulnerable, se relaciona con ésta en términos de objetos a tutelar más que como sujetos con derechos.

En este punto, el análisis del convenio nutre el debate en torno a las condicionales en tanto motoriza nuevos interrogantes: ¿Es legítimo que el Estado bajo el argumento de favorecer el bienestar de la población imponga condicionalidades que plantean exigencias no previstas para el resto de la población? ¿Cuáles son los supuestos y consideraciones sobre los receptores de la AUH que permitieron que se operacionalice el convenio con lo que el mismo implica?

Ciertas lógicas justificatorias de las condicionalidades aparecen con sus especificidades en el caso bajo análisis: la idea de que los pobres deben dar "algo" a cambio de lo que reciben; y que el límite de lo que se puede exigir se define exclusivamente por el criterio de quienes diseñan y aprueban la política social. La idea implícita es que "si el Estado gasta dinero en garantizar derechos sociales, éste se encuentra legitimado para pedir algo a cambio" (AZRAK; ORLANDO, 2013, p. 116).

Esta lógica presenta dos cuestiones sobre los que interesa alertar: la primera, que ya ha sido abordada en la literatura especializada que adscribe al Enfoque de Derechos, es que el Estado a través de las transferencias no esta realizando una concesión benéfica a las familias, sino que cumpliendo derechos que tiene obligación de satisfacer. Es decir que, la lógica del intercambio, en función de la cual como se transfiere ingresos se estaría legitimado para postular exigencias a cambio, es errónea. Lo dicho no niega que los ciudadanos tengan obligaciones ante la comunidad (y no sólo derechos), sino que lo que sostiene es que esas obligaciones son universales e independientes de las estrategias que el Estado implemente para garantizar Derechos.

La segunda cuestión, es que tanto la $\mathrm{AUH}$, cuentan con un importante número de receptores y bases nominadas. Ello se convierte en un gran potencial para identificar y evaluar la situación socioeconómica de grupos poblacionales. Estas cualidades han llevado a conceptualizar a políticas de éste tipo (amplio alcance y bases) como "puertas de entrada a la protección Social" (CECCHINI; MARTÍNEZ, 2010, p. 133) al permitir el paso de las familias e individuos a otros programas. Estas potencialidades cuando son puestas en juego bajo la lógica justificatoria del intercambio, corren el riesgo de que se utilicen como perchas donde se cuelga "por el mismo precio" nuevas exigencias y objetivos, como ocurre en el caso bajo análisis.

El caso en estudio, a su vez, presenta una particularidad que vale la pena mencionar: el convenio en primer término supone la encuesta que tiene como objeto la producción de información pública y luego, recién en segundo lugar, en los casos que corresponda, la realización de los controles médicos para los receptores en riesgo. Es decir, el caso adquiere una originalidad mayor en función que el primer objetivo del convenio supone colgar nuevas exigencias a los receptores de la AUH para suplir las carencias del Estado en materia de información, y luego, recién en segundo término, aparecería la condicionalidad con su objetivo de supervisar la salud de los receptores. 


\section{Palabras finales}

A lo largo de este capítulo se pudo observar, como el análisis de un convenio de colaboración entre dos organismos que regula una intervención tan específica como la realización de una encuesta socio-ambiental en el territorio de la CMR, da pie para el estudio de dos dispositivos relevantes en materia de políticas públicas. Por un lado, las instancias de relevamiento y producción de información, y por el otro, los usos de las políticas sociales y sus condicionalidades.

El análisis arrojó las siguientes conclusiones: el convenio es una herramienta cuyo objetivo principal es cumplir con los requerimientos de la Corte en materia de producción de información ordenados en la causa "Mendoza". La lectura cronológica de los fallos que componen esta causa específica deja entrever cuáles son los principales déficits informacionales del Estado a lo largo del abordaje de una problemática ambiental. Aunque basados en el estudio de un caso particular, estos déficits constituyen una guía por donde empezar a estudiar de forma más general, cómo opera en otros casos aquello que aquí se denominó el componente informacional del proceso de formulación de políticas públicas.

Los déficits en materia de producción de información salen a la luz, tanto al momento de elaborar la demanda que originó la causa "Mendoza" como también en el Informe de Peritaje de la UBA, realizado a fin de evaluar el Plan Integral que presentó el PEN a pedido de la Corte. El Plan Integral no incluye momentos de relevamiento de información y análisis de datos dentro del proceso de diseño, ejecución e implementación de la política o programa cristalizado en el Plan. La mayoría de las debilidades iluminadas en dicho informe van directo hacia el corazón del componente informacional: el Plan no prevé instrumentos ni instancias de producción de información que permitan identificar problemas prioritarios, definir objetivos y evaluar su consecución a través de indicadores pertinentes. Por el contrario, los datos se relevan por fuera del proceso de formulación, ex post, y en general con el objeto de intentar evaluar los resultados de la política. Es decir que no sólo existían déficits informacionales ex ante, que se ponen en evidencia en la causa, sino que una vez que estos son detectados y explicitados a través de los fallos de la Corte, el Plan que se produce vuelve a repetir las mismas fallas.

El convenio Anses-Acumar contrasta con las otras intervenciones del mismo organismo ya citadas (Eisar), donde las familias sobre las que se aplicaron los estudios sí tuvieron la instancia de consentir su participación, a diferencia de lo previsto en el convenio. Esto se debe a que, como se vio, subyace en las políticas sociales destinadas a población calificada como vulnerable la idea de que quienes reciben prestaciones sociales, deben devolverle algo a la sociedad; desde esta mirada a los receptores se les puede sumar exigencias sin límite preestablecido. Más allá, de que la encuesta per se estudiada en este caso no aparece como una carga desmedida sobre los receptores, lo que interesa es advertir sobre la naturalización de estas prácticas y poner en evidencia los supuestos que se encuentran detrás de la misma.

\section{Referencias}

ACUMAR-Autoridad de Cuenca Matanza Riachuelo. La Acumar realiza una evaluación de salud en el barrio Acuba. 19 dic. 2012. Disponible en: <http://www.acumar.gov.ar/noved_noticias_pdf.php?num=422>. Acceso en: 3 jul. 2012.

La Acumar realiza una evaluación de salud en la Villa 21-24. 3 ago. 2012. Disponible en: <http://www.acumar.gov.ar/ noved_noticias_pdf.php?num=373>. Acceso en: 3 jul. 2012.

. Hacemos tareas de prevención en las zonas de riesgo de la cuenca. 24 jul. 2012. Disponible en: <http://www.acumar.gov.ar/ noved_noticias_pdf.php?num=369>. Acceso en: 3 jul. 2012.

ARGENTINA. Convenio Anses-Acumar. Buenos Aires, Argentina, 2012.

Ley n. 26.168, de 15 de noviembre de 2006. Crea la Autoridad de la Cuenca Matanza Riachuelo. Disponible en: <http:// www.infoleg.gov.ar/infolegInternet/anexos/120000-124999/122769/norma.htm>. Acceso en: 8 abr. 2013.

AZRAK, D.; ORLANDO, F. Las condicionalidades en los Programas de Transferencia de Ingresos: aportes desde una lectura igualitaria de la Constitución. En: BERCOVICH, L.; MAURINIO, G. (Coord.). Los derechos sociales en la Gran Buenos Aires. Algunas aproximaciones desde la teoría, las instituciones y la acción. Buenos Aires: Eudeba, 2013, p. 103-122.

CECCHINI, S.; MADARIAGA, A. La trayectoria de los programas de transferencias con corresponsabilidad (PTC) en América Latina y el Caribe. Santiago de Chile: División de Desarrollo Social. Comisión Económica para América Latina y el Caribe, Cuaderno de la CEPAL, n. 95, 2011.

CECCHINI, S.; MARTÍNEZ, R., 2010. Protección social inclusiva en América Latina. Una mirada integral, un enfoque de derechos. Santiago de Chile: Comisión Económica para América Latina y el Caribe (CEPAL), Libro de la CEPAL, n. 111, 2011.

CEPAL-Comisión Económica para América Latina y el Caribe. Programas de Transferencias Condicionadas: Base de datos de programas de protección social no contributiva en América Latina y el Caribe. Disponible en: 〈http://dds.cepal.org/bdptc/>. Acceso en: 3 jul. 2012. 
FALLO "Mendoza, Beatriz Silvia y otros c/Estado Nacional y otros s/daños y perjuicios (daños derivados de la contaminación ambiental del Río Matanza-Riachuelo)" (M.1569.XL) - Corte Suprema de Justicia de la Nación de fecha 20 de Junio de 2006. FALLO "Mendoza, Beatriz Silvia y otros c/Estado Nacional y otros s/daños y perjuicios (daños derivados de la contaminación ambiental del Río Matanza-Riachuelo)" (M.1569.XL) - Corte Suprema de Justicia de la Nación de fecha 22 de agosto de 2007. FALLO "Mendoza, Beatriz Silvia y otros c/Estado Nacional y otros s/daños y perjuicios (daños derivados de la contaminación ambiental del Río Matanza-Riachuelo)” (M.1569.XL) - Corte Suprema de Justicia de la Nación de fecha 08 de Julio de 2008. FALLO "Mendoza, Beatriz Silvia y otros c/Estado Nacional y otros s/daños y perjuicios (daños derivados de la contaminación ambiental del Río Matanza-Riachuelo)" (M.1569.XL) - Corte Suprema de Justicia de la Nación de fecha 10 de agosto de 2010. UNODC-Oficina de Naciones Unidas Contra la Droga y el Delito. Informe mundial sobre drogas 2013. Viena, 26 jun. 2013. SIGAL, M.; BECÚ, N. Los datos para la igualdad educativa. La Nación, 2013. Disponible en: <http://www.lanacion.com.ar/947050datos-para-la-igualdad-educativa>. Acceso en: 5 ago. 2013.

UBA-Universidad de Buenos Aires. Informe de avance Cuenca Matanza-Riachuelo, 2007. Disponible en: <http://www.farn.org.ar/ participacion/riachuelo/documentos/informe_uba.pdf>. Acceso en: 5 ago. 2013.

\section{Notas}

1 La Autoridad de Cuenca Matanza Riachuelo (Acumar), es un organismo público que se desempeña como la máxima autoridad en materia ambiental en la región. Es un ente autónomo, autárquico e interjurisdiccional que conjuga el trabajo con los tres gobiernos que tienen competencia en el territorio: Nación, Provincia de Buenos Aires y Ciudad Autónoma de Buenos Aires.

2 El sistema de Asignaciones Familiares Nacionales (Ley 24-714) se encuentra actualmente conformado por 3 subsistemas: uno contributivo destinado a los trabajadores formales y receptores del seguro de desempleo; uno no contributivo destinado a los trabajadores pasivos (que perciban jubilación y/o pensión) y, finalmente, un tercer subsistema no contributivo creado a partir de la AUH - Decreto1602/09 -, destinado a los trabajadores informales, desocupados, trabajadoras del servicio doméstico y monotributistas sociales. A estas vías, se le suman las deducciones al impuesto a las ganancias que se realizan por hijos, cónyuges, y familiares directos que implican una transferencia implícita del Estado hacia las familias de mayores recursos.

3 La inexistencia de datos públicos en la Argentina no es exclusiva de la problemática ambiental. En el área de educación, por ejemplo, laAsociación Civil por la Igualdad (ACIJ), especializada en la temática, señaló en varias ocasiones la falta de producción, sistematización y centralización de datos estadísticos en ese campo. Ello constituye un obstáculo para el diseño de políticas que logren redistribuir recursos en pos de la igualdad (SIGAL; BECÚ, 2013). En materia de seguridad ciudadana ocurre algo similar. A modo de ejemplo se cita un informe de la ONU, del año 2011, que advierte sobre la falta de información oficial por parte de la Argentina respecto de los secuestros de drogas (UNODC, 2013).

4 La norma que regula en la Argentina el derecho fundamental de todas las personas a acceder a información en manos del Estado es el Decreto $\mathrm{N}^{\circ}$ 1172, dictado en el año 2003 por el Poder Ejecutivo Nacional. El contar con una ley nacional de acceso a la información que abarque a los tres poderes del Estado es aún una cuenta pendiente. Para la elaboración de este artículo se enviaron pedidos de información enmarcados en el citado Decreto a la Acumar, la Secretaria de Ambiente de la Nación y a la Anses. Los primeros dos organismos no contestaron los requerimientos de información incumpliendo con la norma.

5 La vinculación entre las exigencias de la Corte y la estrategia implementada por laAcumar, a través del acuerdo con Anses, queda explicitada en los considerandos de tal convenio: Que a partir de la sentencia de la Corte Suprema de Justicia de la Nación en el marco de la Causa "Mendoza Beatriz, y otros c/Estado Nacional y otros s/daños y perjuicios (daños derivados de la Contaminación ambiental del Río Matanza-Riachuelo (ARGENTINA. ConvenioANSES-ACUMAR, de julio de 2012, p. 1) se determinó la obligación de la Autoridad de la Cuenca Matanza Riachuelo de implementar un Plan Sanitario de Emergencia que contemple, entre otras cosas, la determinación de la población en situación de riesgo y la puesta en marcha de programas sanitarios específicos destinados a satisfacer las demandas de la población en materia de salud (...)”.

6 El reconocimiento del acceso a la información como derecho humano ha ido evolucionando progresivamente en el marco del derecho internacional de los derechos humanos. El Sistema Interamericano de Derechos Humanos ha cumplido en ello un rol fundamental. En el año 2006 la Corte Interamericana de Derechos Humanos, en el caso Claude Reyes y otros, marcó un hito jurisprudencial al constituirse en el primer Tribunal Internacional en reconocer que el acceso a la información es un derecho humano que forma parte del derecho a la libertad de expresión.

7 El documento "Programas de Transferencias Condicionadas. Balance de la experiencia reciente en América Latina y el Caribe", constituye una de las pocas excepciones de lo sostenido. En el mismo las condicionalidades son definidas como "los compromisos concretos que las familias tienen que cumplir para recibir las transferencias" (CECCHINI; MADARIAGA, 2011, p. 10).

\section{Mora Straschnoy}

mora@straschnoy.com

Maestranda en Políticas Sociales en la Universidad de Buenos Aires (UBA)

Docente en la UBA

Becaria Conicet. 


\section{Mora Kantor}

kantor.mora@gmail.com

Maestranda en Administración y Políticas Públicas por la Universidad de San Andrés

Docente en la UBA

\section{UBA - Universidad de Buenos Aires}

Av. Figueroa Alcorta 2263

CEP: C1425CKB 